\title{
Glucagon-Like Peptide-1 Receptor Agonist Utilization in Type 2 Diabetes in Japan: A Retrospective Database Analysis (JDDM 57)
}

\author{
Yasushi Ishigaki · Alena Strizek · Toshihiko Aranishi (D) • \\ Nobuhiro Arai $\cdot$ Takeshi Imaoka $\cdot$ Zhihong Cai $\cdot$ Hiroshi Maegawa
}

Received: August 17, 2020 / Accepted: November 24, 2020 / Published online: December 9, 2020

(C) The Author(s) 2020

\section{ABSTRACT}

Introduction: There are limited real-world data on the prescribing of glucagon-like peptide-1 receptor agonists (GLP-1 RAs) for patients with type 2 diabetes mellitus (T2DM).

Methods: This was a retrospective analysis of the CoDiC ${ }^{\circledR}$ database of the Japan Diabetes Clinical Data Management Study Group (JDDM). Demographic and clinical characteristics, concomitant treatment patterns, and GLP1 RA treatment persistence or modification in

Supplementary Information The online version of this article (https://doi.org/10.1007/s13300-020-00977w) contains supplementary material, which is available to authorized users.

Y. Ishigaki

Division of Diabetes, Metabolism and

Endocrinology, Iwate Medical University, Morioka, Japan

A. Strizek

Eli Lilly and Company, Sydney, Australia

T. Aranishi $(\bowtie) \cdot$ T. Imaoka $\cdot$ Z. Cai

Eli Lilly Japan K.K., Kobe, Japan

e-mail: aranishi_toshihiko@lilly.com

N. Arai

Deloitte Tohmatsu Consulting LLC, Tokyo, Japan

H. Maegawa

Department of Medicine, Shiga University of

Medical Science, Shiga, Japan patients with T2DM initiating GLP-1 RA therapy were evaluated.

Results: The analysis included 932 eligible patients with T2DM who had their first GLP-1 RA prescription (index date) between September 2016 and July 2018. Mean age was 63.8 years and $56.0 \%$ were male. Most patients had an index GLP-1 RA of dulaglutide (65.7\%) or liraglutide (29.1\%). Common comorbidities were obesity $(58.7 \%)$, hypertension $(54.7 \%)$, dyslipidemia $(52.0 \%)$, retinopathy $(11.3 \%)$, and nephropathy (10.2\%). Mean hemoglobin A1c (HbA1c) levels decreased from 8.3 to $7.8 \%$ over 6 months after GLP-1 RA initiation, and the proportion of patients achieving $\mathrm{HbA} 1 \mathrm{c}<7.0 \%$ increased from $14.4 \%$ at index date to $22.9 \%$ at 6 months. Reductions occurred in mean body weight, body mass index, triglycerides, total cholesterol, lowdensity lipoprotein cholesterol, and adjusted urinary albumin over 6 months. Antidiabetic medication use decreased after GLP-1 RA initiation, whereas non-antidiabetic medication prescribing showed little change. Index GLP-1 RA persistence rates were $80.5 \%, 66.2 \%$, and $51.6 \%$ at 6,12 , and 18 months post-index, respectively, with a median persistence until discontinuation or switch of 600 days. Persistence rates at 6, 12, and 18 months post-index, respectively, were $81.9 \%, 70.7 \%$, and $65.4 \%$ for dulaglutide and $79.7 \%, 60.0 \%$, and $30.4 \%$ for liraglutide.

Conclusion: The study shows real-world benefits of GLP-1 RA therapy for T2DM, including improvements in HbA1c, body weight, and 
blood lipid profile, and supports the high rates of long-term persistence previously reported with dulaglutide, the GLP-1 RA most commonly prescribed for T2DM in Japanese clinical practice.

\section{PLAIN LANGUAGE SUMMARY}

Glucagon-like peptide-1 receptor agonists (GLP1 RAs) are drugs that patients with type 2 diabetes mellitus (T2DM) take to help control their blood sugar levels. In Japan, the GLP-1 RAs that doctors can prescribe are dulaglutide, exenatide, liraglutide, and lixisenatide as of May 2020. We conducted a study of how GLP-1 RAs are used to treat patients with T2DM in Japanese real-world clinical practice. We used a large database of anonymous information from hospitals and clinics in Japan. Over 900 adult patients started their first GLP-1 RA treatment for T2DM between September 2016 and July 2018. Before these patients started GLP-1 RA treatment, many were overweight, had high blood pressure, and had abnormal levels of lipids in their blood. Six months after starting GLP-1 RA treatment, on average these patients had lower hemoglobin A1c (a measure of average blood sugar levels), lower body weight, and better blood lipid levels than before they started GLP-1 RA treatment. Dulaglutide was the most common GLP-1 RA prescribed, then liraglutide. After 6 months, most patients (four-fifths) continued to use their GLP-1 RA treatment without stopping or changing to another treatment. After 18 months, half of the patients were still using their GLP-1 RA. Two-thirds of patients on dulaglutide and onethird of patients on liraglutide continued the treatment after 18 months. This study shows that GLP-1 RAs can benefit patients with T2DM in real-world clinical practice. It also shows that patients may be able to take long-term dulaglutide treatment.

Keywords: Diabetes mellitus, type 2; Glucagon-like peptide-1 receptor agonist; Registry; Treatment persistence

\section{Key Summary Points}

\section{Why carry out this study?}

There are limited data on the prescribing of glucagon-like peptide- 1 receptor agonists (GLP-1 RAs) for patients with type 2 diabetes mellitus (T2DM) in Japanese clinical practice.

\section{What was learned from the study?}

Patients initiating GLP-1 RA therapy commonly have comorbid obesity (58.7\%), hypertension (54.7\%), and dyslipidemia (52.0\%).

GLP-1 RA therapy was associated with improvements in hemoglobin A1c, body weight, and blood lipid profile at 6 months after patients initiated treatment.

Index GLP-1 RA persistence rates were $80.5 \%, 66.2 \%$, and $51.6 \%$ at 6,12 , and 18 months post-index, respectively, with a median persistence until discontinuation or switch of 600 days.

Dulaglutide, the most commonly prescribed GLP-1 RA (65.7\%), showed persistence rates at 12 and 18 months of $70.7 \%$ and $65.4 \%$, respectively, and liraglutide showed persistence rates of $60.0 \%$ and $30.4 \%$, respectively.

\section{DIGITAL FEATURES}

This article is published with digital features to facilitate comprehension of the article. To view digital features for this article, go to https://doi. org/10.6084/m9.figshare.13281140.

\section{INTRODUCTION}

An estimated $13.6 \%$ of the Japanese adult population are strongly suspected of having diabetes mellitus [1]. The prevalence of strongly 
suspected diabetes mellitus has almost doubled over the past 20 years in Japanese males, from $9.9 \%$ in 1997 to $18.1 \%$ in 2017, and has increased in females from 7.1 to $10.5 \%[1,2]$. Clinical guidelines published by the Japan Diabetes Society recommend oral antidiabetic medications and/or injectable glucagon-like peptide-1 receptor agonists (GLP-1 RAs) or insulin for non-insulin-dependent patients with diabetes that is not controlled by diet and exercise, and combination therapy of insulin plus oral antidiabetic medications or GLP-1 RAs in diabetic patients requiring intensive insulin therapy [3]. However, unlike clinical guidelines from the United States (US) or Europe [4], Japanese guidelines do not recommend specific first-line or combination antihyperglycemic agents from the available treatment options [3]. Consequently, the choice of diabetes medication prescribed in Japan depends predominantly on physician judgment and preference.

Previous studies have identified differences in prescribing patterns between Japan and the US or Europe for patients with type 2 diabetes mellitus (T2DM), such as earlier and more frequent use of dipeptidyl peptidase-4 (DPP-4) inhibitors in Japan $[5,6]$. These variations in treatment practices may have arisen because Asian patients with T2DM, including Japanese, are characterized to a greater extent by impaired insulin secretion rather than by increased insulin resistance when compared with Caucasian patients [7]. Additionally, because of increases in obesity among Japanese diabetic patients, Japanese physicians may prefer to prescribe treatments that are not associated with weight increase or that promote weight loss [8]. To date, there has been insufficient evidence in Japanese patients with T2DM to determine the most appropriate first-line or second-line antihyperglycemic medications [9].

GLP-1 RAs, also known as incretin mimetics, are a class of injectable antidiabetic drugs that stimulate glucose-dependent insulin secretion, suppress postprandial glucagon secretion, and induce satiety by slowing gastric emptying, leading to weight loss [10-12]. In Japan, GLP-1 RAs have been available for 10 years, with the approval of liraglutide and exenatide in 2010, a once-weekly regimen of exenatide in 2012, lixisenatide in 2013, and dulaglutide in 2015. Despite GLP-1 RAs showing greater efficacy in Asian versus non-Asian populations [13], and their beneficial effects on weight loss [10], the use of GLP-1 RAs in Japan is low [14]. Although GLP-1 RAs are recommended treatment options for patients with T2DM in the Japan Diabetes Society guidelines [3], there are limited data on the prescribing of GLP-1 RAs in Japanese clinical practice.

The aim of this retrospective analysis of Japanese diabetes registry data was to understand GLP-1 RA use in patients with T2DM by assessing the demographic and clinical characteristics of patients initiating GLP-1 RA therapy. The study also assessed concomitant treatment patterns of patients initiating GLP-1 RA therapy and GLP-1 RA treatment persistence or modification in this Japanese real-world clinical setting.

\section{METHODS}

\section{Study Design}

This retrospective observational study analyzed data from the Computerized Diabetes Care database (CoDiC $\AA)$ of the Japan Diabetes Clinical Data Management Study Group (JDDM). This database was specifically developed by the JDDM to promote research on medical care for diabetes in Japan [15]. CoDiC is a large validated database containing anonymized, longitudinal, patient-level information, including diagnoses, laboratory results, and prescription data. The database holds records for approximately 60,000 patients from 98 institutions specializing in diabetes treatment across Japan and is updated annually.

The study protocol was approved by the JDDM Ethical Review Board (JDDM2018-2). All patients provided informed consent. The study was conducted in accordance with the ethical principles of the Declaration of Helsinki 1964 and its later amendments, Good Pharmacoepidemiology Practices, and applicable local laws and regulations. 


\section{Patient Selection}

The target population was adult Japanese patients with T2DM who were identified as new users of GLP-1 RA therapy based on the date of their first GLP-1 RA prescription (the index date) in CoDiC database records. Eligible patients were those with an index date (first GLP-1 RA prescription date) between September 1, 2016 and July 31,2018 , who had been previously diagnosed with T2DM and were $\geq 18$ years on their index date. The index date window of September 2016 to July 2018 was chosen to commence once prescribing restrictions were consistent for all GLP-1 RAs, as new pharmaceutical products in Japan are generally restricted to 14-day prescriptions for the first year. Patients were to have follow-up data, defined as evidence of prescription activity for any drug, for $\geq 6$ months before and $\geq 6$ months after their index date. To capture $\geq 6$ months of data either side of the index date window, the analysis included CoDiC data from March 1, 2016 to January 31, 2019.

Patients were excluded if their records for the 6 months before the index date showed previous GLP-1 RA use; if they were prescribed any GLP-1 RA therapy for weight loss; had type 1 or gestational diabetes; or had undergone bariatric surgery. GLP-1 RA prescriptions were identified by searching for the five GLP-1 RA regimens that were approved and commercially available in Japan: liraglutide, exenatide twice daily, exenatide once weekly, lixisenatide, and dulaglutide.

\section{Outcome Measures}

Patient information obtained from the CoDiC database included age at index date, sex, duration of T2DM from onset to index date, and comorbidities (predefined as nephropathy, retinopathy, myocardial infarction, cerebral infarction, hypertension, dyslipidemia, lower extremity amputations, and obesity, defined as body mass index $[\mathrm{BMI}] \geq 25 \mathrm{~kg} / \mathrm{m}^{2}$ ). Comorbid hypertension and dyslipidemia were identified by prescriptions for antihypertensive or lipidlowering treatments, respectively, and other comorbidities were identified directly from database records.

Clinical characteristics data included hemoglobin A1c (HbA1c), weight, BMI, triglycerides (TG), total cholesterol (TC), high-density lipoprotein cholesterol (HDL-C), low-density lipoprotein cholesterol (LDL-C), serum creatinine, and urinary albumin adjusted for creatinine. Available data for clinical characteristics were identified at the index date, using the closest available assessment to the index date in a window of -183 days to +14 days, and also at 6 months (183 days) follow-up, using the closest available assessment in a window of +93 days to +228 days. An exploratory analysis of $\mathrm{HbA} 1 \mathrm{c}$ at index date and at 6 months follow-up was performed in the subpopulation of GLP-1 RA continuers, defined as patients who continued their index GLP-1 RA therapy for the 6-month post-index period. The subpopulation of GLP-1 RA continuers comprised $80.5 \%$ of the GLP-1 RA population. Changes in clinical characteristics from index date to 6 months after GLP-1 RA initiation were calculated for HbA1c, weight, and BMI. The proportions of patients with HbA1c levels of $<6.0 \%, \geq 6.0 \%$ to $<7.0 \%, \geq 7.0 \%$ to $<8.0 \%$, and $\geq 8.0 \%$ at index date and at 6 months follow-up were calculated.

Medication use was identified from the database records based on prescription data. Medications for the 6-month pre-index period were those prescribed during a window of -183 days to -1 day before the index date. Concomitant medications during the 6-month post-index period included medications prescribed at the index date and medications that were prescribed during both the 6-month pre-index period and the post-index period (index date to the index GLP-1 RA discontinuation or switch date or +183 days, whichever came first), with an interval between consecutive prescriptions of $\leq 90$ days that overlapped with the index date. GLP-1 RA prescriptions were categorized by individual product. Antidiabetic medications were categorized by product class (alpha-glucosidase inhibitors, biguanides, DPP-4 inhibitors, glinides, sodium-glucose transport protein 2 [SGLT2] inhibitors, sulfonylureas, thiazolidinediones, and prandial, basal, and premix 
insulins), and non-antidiabetic medications were categorized by therapy class (antiplatelet medication, antihypertensive medication, and lipid-lowering medication).

Index GLP-1 RA treatment modifications were classified as discontinuation, switch, or augmentation, and were assessed using overall follow-up data at 6,12 , and 18 months postindex. As physicians in Japan prescribe sufficient medication to cover the duration between visits and patients make an average of 12 visits to specialists per year [16], discontinuation was defined as an absence of any prescription for the index GLP-1 RA for $>60$ days since the previous visit. Switch was defined as the prescription of a new antidiabetic medication within 30 days before or after discontinuation of the index GLP-1 RA. Augmentation was defined as the prescription of a new antidiabetic medication $>30$ days before index GLP-1 RA discontinuation or the end of the follow-up period, whichever came first. Time to treatment modifications of the index GLP-1 RA was evaluated, as well as persistence, which was defined as the duration of index GLP-1 RA therapy from initiation to discontinuation or switch.

\section{Statistical Analysis}

Analyses were conducted for the GLP-1 RA population, which included all eligible patients identified from the database, and for the subgroups of patients with an index GLP-1 RA of dulaglutide or liraglutide. The proportions of patients with an index GLP-1 RA of lixisenatide, exenatide twice daily, or exenatide once weekly were insufficient for separate by-treatment analyses. Patient demographic and clinical characteristics, medication use, and GLP-1 RA treatment persistence and modifications were summarized descriptively. Persistence was described using Kaplan-Meier survival analysis. Missing data were not imputed for any outcome measures. Statistical analysis was performed by Deloitte Tohmatsu Consulting LLC using $\mathrm{R}$ version 3.6.1 ( $\mathrm{R}$ Foundation for Statistical Computing, Vienna, Austria).

\section{RESULTS}

\section{Patient Population and Characteristics}

A total of 932 patients with T2DM met the eligibility criteria and were included in the GLP-1 RA population (Fig. 1). The majority of the patients had an index GLP-1 RA therapy of dulaglutide (612 patients; $65.7 \%$ ) or liraglutide (271 patients; $29.1 \%$ ). The remaining patients were prescribed lixisenatide (38 patients; $4.1 \%$ ), exenatide twice daily (6 patients; $0.6 \%$ ), or exenatide once weekly ( 5 patients; $0.5 \%$ ) as the index GLP-1 RA. Among the GLP-1 RA population, 750 patients $(80.5 \%)$ continued to take their index GLP-1 RA therapy for $\geq 6$ months and were included in the GLP-1 RA continuer subpopulation (Fig. 1).

The mean age in the GLP-1 RA population was 63.8 years, and $56.0 \%$ of patients were male (Table 1). The mean duration of diabetes was

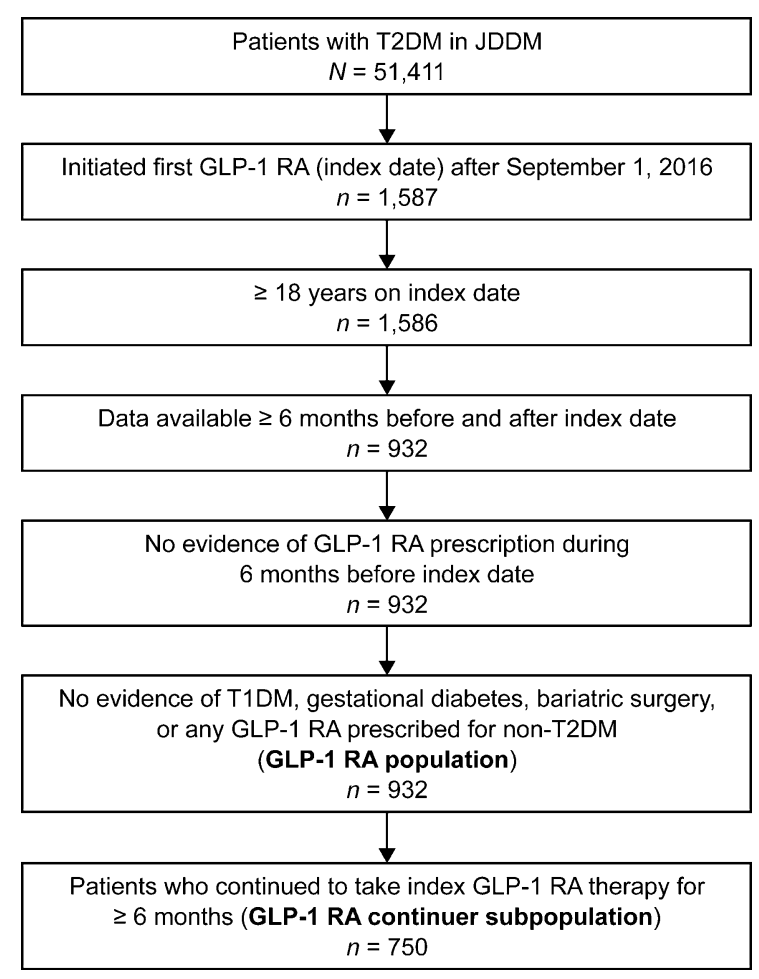

Fig. 1 Patient identification. GLP-1 $R A$ glucagon-like peptide-1 receptor agonist, $J D D M$ Japan Diabetes Clinical Data Management Study Group, TIDM type 1 diabetes mellitus, $T 2 D M$ type 2 diabetes mellitus 
Table 1 Patient characteristics

\begin{tabular}{|c|c|c|c|}
\hline Variable & All GLP-1 RA $(n=932)$ & Dulaglutide $(n=612)$ & Liraglutide $(n=271)$ \\
\hline Age, years & $63.8(13.3)$ & $64.6(13.3)$ & $63.4(12.8)$ \\
\hline \multicolumn{4}{|l|}{ Age category, years, $n(\%)$} \\
\hline $18-29$ & $9(1.0)$ & $5(0.8)$ & $3(1.1)$ \\
\hline $30-39$ & $32(3.4)$ & $17(2.8)$ & $11(4.1)$ \\
\hline $40-49$ & $110(11.8)$ & $67(10.9)$ & $29(10.7)$ \\
\hline $50-59$ & $168(18.0)$ & $112(18.3)$ & $44(16.2)$ \\
\hline $60-69$ & $275(29.5)$ & $177(28.9)$ & $90(33.2)$ \\
\hline $70-79$ & $226(24.2)$ & $142(23.2)$ & $74(27.3)$ \\
\hline$\geq 80$ & $112(12.0)$ & $92(15.0)$ & $20(7.4)$ \\
\hline Male, $n(\%)$ & $522(56.0)$ & $329(53.8)$ & $171(63.1)$ \\
\hline Body weight, $\mathrm{kg}^{\mathrm{a}}$ & $71.7(17.9)$ & $71.1(17.6)$ & $71.6(17.5)$ \\
\hline BMI, $\mathrm{kg} / \mathrm{m}^{2 \mathrm{~b}}$ & $27.4(5.4)$ & $27.5(5.4)$ & $26.8(5.3)$ \\
\hline Duration of diabetes, years ${ }^{c}$ & $16.1(9.6)$ & $16.2(9.6)$ & $16.5(9.5)$ \\
\hline \multicolumn{4}{|l|}{ Comorbidities, $n(\%)$} \\
\hline Obesity & $547(58.7)$ & $367(60.0)$ & $140(51.7)$ \\
\hline Hypertension & $510(54.7)$ & $326(53.3)$ & $163(60.1)$ \\
\hline Dyslipidemia & $485(52.0)$ & $317(51.8)$ & $144(53.1)$ \\
\hline Retinopathy & $105(11.3)$ & $55(9.0)$ & $42(15.5)$ \\
\hline Nephropathy & $95(10.2)$ & $76(12.4)$ & $15(5.5)$ \\
\hline Myocardial infarction & $2(0.2)$ & $1(0.2)$ & $1(0.4)$ \\
\hline Cerebral infarction & $2(0.2)$ & $1(0.2)$ & $1(0.4)$ \\
\hline Lower extremity amputations & 0 & 0 & 0 \\
\hline
\end{tabular}

Data are mean (standard deviation) unless otherwise stated

$B M I$ body mass index, GLP-1 RA glucagon-like peptide-1 receptor agonist

a All GLP-1 RA $n=844$; dulaglutide $n=554$; liraglutide $n=243$

b All GLP-1 RA $n=831$; dulaglutide $n=545$; liraglutide $n=239$

c All GLP-1 RA $n=888$; dulaglutide $n=584$; liraglutide $n=256$

16.1 years, with $45.7 \%$ of patients having had diabetes for $\geq 15$ years. The most common comorbidities were obesity $(58.7 \%)$, hypertension $(54.7 \%)$, dyslipidemia (52.0\%), retinopathy $(11.3 \%)$, and nephropathy (10.2\%) (Table 1).

\section{Change in HbA1c}

At 6 months after GLP-1 RA treatment initiation, mean HbA1c levels decreased from 8.3 to $7.8 \%$ in the GLP-1 RA population (Fig. $2 \mathrm{a}$ ), with $25.3 \%$ of patients experiencing a reduction in HbA1c $>1.0 \%$. The proportion of patients who achieved the target HbA1c $<7.0 \%$ increased 

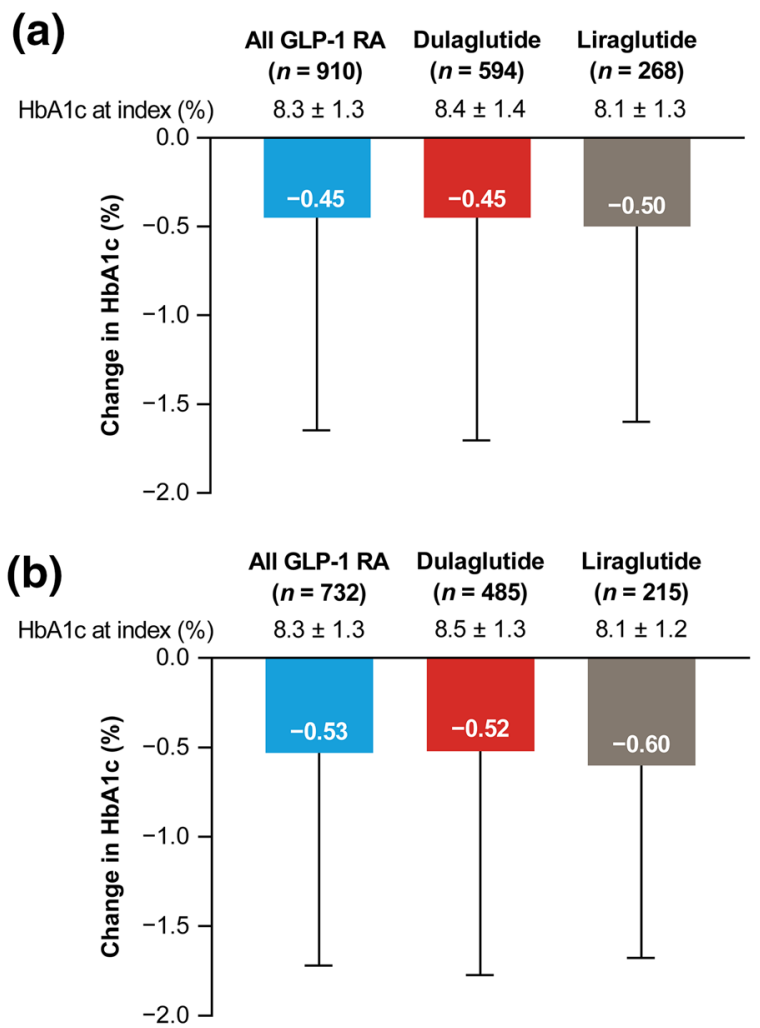

Fig. 2 Change in mean HbAlc from index date to 6 months post-index for a the GLP-1 RA population and b the GLP-1 RA continuers subpopulation (patients who continued on their index GLP-1 RA therapy for $\geq 6$ months). Data are mean \pm standard deviation. Index date includes the closest available HbAlc assessment to the index date in a window of -183 days to +14 days; 6 months post-index includes the closest available HbAlc assessment to 183 days in a window of +93 days to +228 days. GLP-1 RA glucagon-like peptide-1 receptor agonist, $H b A 1 c$ hemoglobin Alc

from $14.4 \%$ at the index date to $22.9 \%$ at 6 months post-index, whereas the proportion of patients with $\mathrm{HbA} 1 \mathrm{c} \geq 8.0 \%$ decreased from 58.0 to $37.7 \%$ (Fig. 3a).

Mean HbA1c levels at the time of treatment initiation were $8.4 \%$ and $8.1 \%$ for patients in the dulaglutide and liraglutide groups, respectively (Fig. 2a). At 6 months after treatment initiation, both the dulaglutide and liraglutide groups showed decreases in mean HbA1c levels of $0.45 \%$ and $0.50 \%$, respectively (Fig. $2 \mathrm{a}$ ). The proportion of patients achieving the target HbA1c (Fig. 3a) increased with dulaglutide and liraglutide and were consistent with the GLP-1 RA population. The proportion of patients with a $>1 \%$ reduction in $\mathrm{HbA} 1 \mathrm{c}$ was $26.8 \%$ in the dulaglutide group and $23.5 \%$ in the liraglutide group.

\section{GLP-1 RA Continuer Analysis of Change in HbA1c}

In the GLP-1 RA continuer subpopulation, which included all patients who continued on their index GLP-1 RA therapy for $\geq 6$ months, the reduction in mean $\mathrm{HbA} 1 \mathrm{c}$ from index date to 6 months post-index (0.53\%; Fig. $2 \mathrm{~b})$ was consistent with the overall GLP-1 RA population (0.45\%; Fig. 2a). The proportion of patients with a reduction in $\mathrm{HbA} 1 \mathrm{c}$ of $>1.0 \%$ was also similar for the GLP-1 RA continuers (27.7\%) and the overall GLP-1 RA population (25.3\%). The target $\mathrm{HbA} 1 \mathrm{c}$ of $<7.0 \%$ was achieved by $13.1 \%$ of GLP-1 RA continuers at the index date, increasing to $23.4 \%$ at 6 months post-index, whereas the proportion of continuers with HbA1c $\geq 8.0 \%$ decreased from $59.3 \%$ to $36.3 \%$ over the 6-month post-index period (Fig. 3b).

Reductions in mean HbA1c from index to 6 months post-index were $0.52 \%$ and $0.60 \%$ in GLP-1 RA continuers receiving dulaglutide and liraglutide, respectively (Fig. 2b), and a reduction in HbA1c of $>1.0 \%$ was achieved in $29.1 \%$ of dulaglutide continuers and $26.0 \%$ of liraglutide continuers. Improvements in target HbA1c achievement (Fig. 3b) seen after 6 months of treatment with dulaglutide or liraglutide were consistent with those seen in the GLP-1 RA population (Fig. 3a).

\section{Other Clinical Characteristics}

During the 6-month period after initiating GLP$1 \mathrm{RA}$, patient clinical characteristics in the GLP1 RA population generally showed clinically meaningful improvement. Mean body weight decreased by $1.3 \mathrm{~kg}$ (Fig. 4f) and mean BMI decreased by $0.5 \mathrm{~kg} / \mathrm{m}^{2}$ (Fig. $4 \mathrm{~g}$ ). Reductions were also seen in mean concentrations of TG (10.8 mg/dL; Fig. 4a), TC (4.7 mg/dL; Fig. 4b), LDL-C (2.2 mg/dL; Fig. $4 d$ ), and adjusted urinary albumin (15.5 mg/dL Cre; Fig. 4 e). There 
(a)

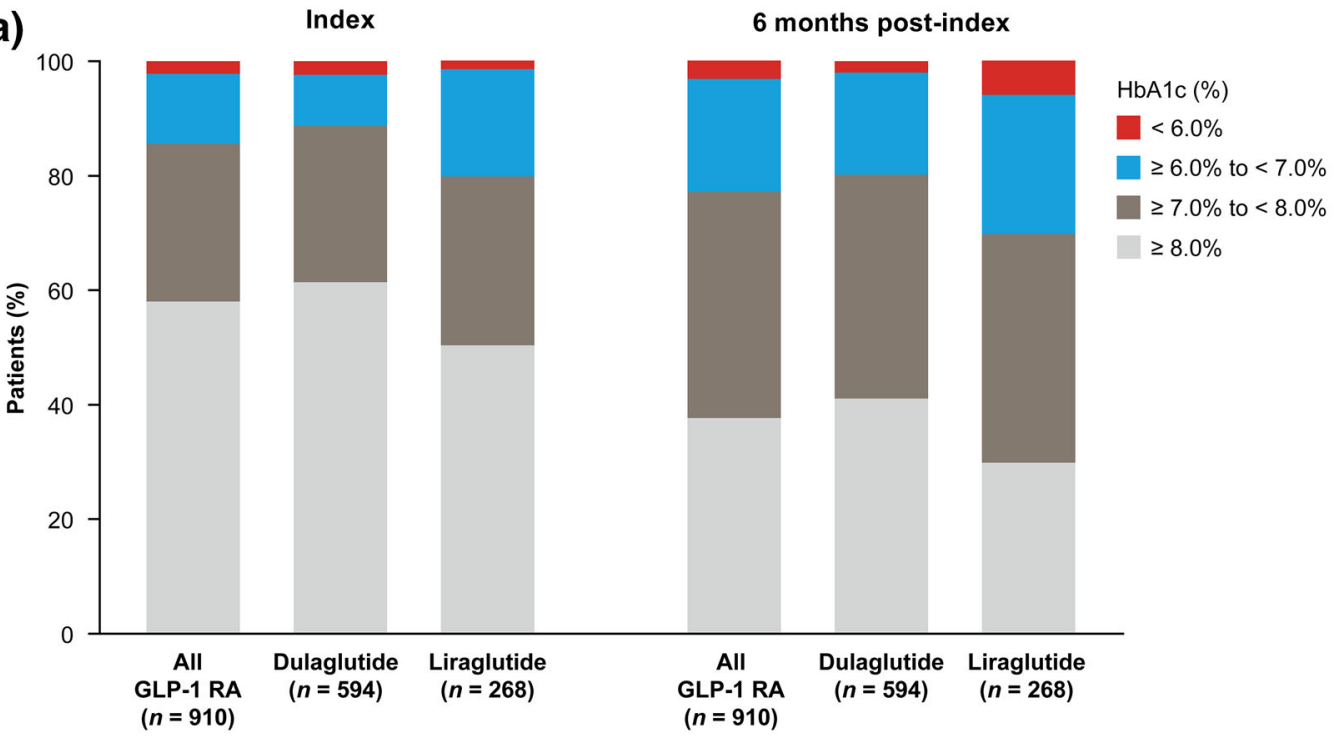

(b)

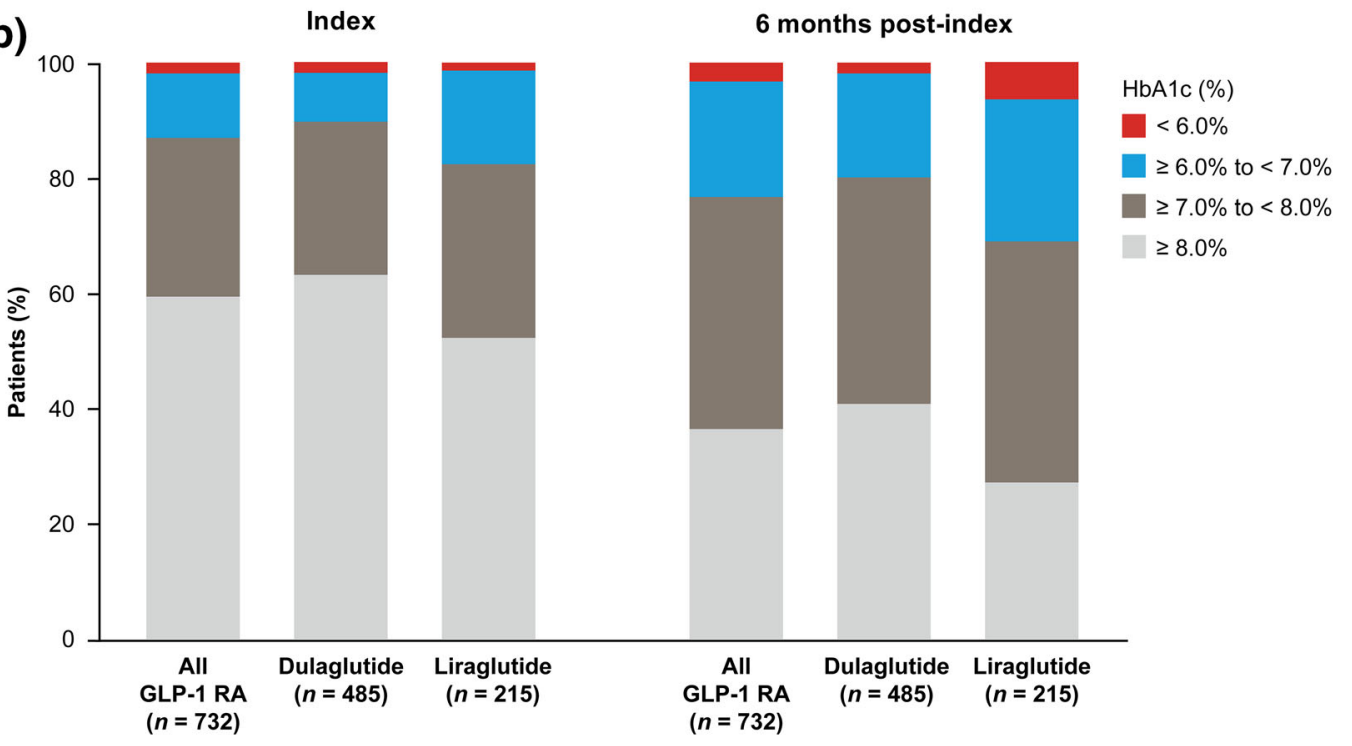

Fig. $3 \mathrm{HbAlc}$ target achievement at index date and 6 months post-index for a the GLP-1 RA population and -183 days to +14 days; 6 months post-index includes the closest available HbAlc assessment to 183 days in a window of +93 days to +228 days. GLP-1 RA glucagon-like peptide-1 receptor agonist, $H b A 1 c$ hemoglobin A1c

continued on their index GLP-1 RA therapy for $\geq 6$ months). Index date includes the closest available $\mathrm{HbA1c}$ assessment to the index date in a window of

was a minimal increase in HDL-C levels $(0.1 \mathrm{mg} / \mathrm{dL}$; Fig. $4 \mathrm{c})$ in the GLP-1 RA population and no change in mean serum creatinine.

The reduction in the mean TG at 6 months post-index for liraglutide was $30.4 \mathrm{mg} / \mathrm{dL}$, while the reduction for dulaglutide was $0.7 \mathrm{mg} / \mathrm{dL}$

(Fig. 4a). Mean HDL-C increased by $0.4 \mathrm{mg} / \mathrm{dL}$ in the dulaglutide group and decreased by $0.6 \mathrm{mg} / \mathrm{dL}$ in the liraglutide group (Fig. 4c); however, the small changes in HDL-C were not considered to be clinically meaningful. 


\section{Antidiabetic Medication}

In the 6 months before initiating GLP-1 RA therapy, $94.0 \%$ of patients in the GLP-1 RA population were prescribed oral antidiabetic medication, with most receiving 2 or 3 classes of antidiabetic medication $(26.9 \%$ and $28.5 \%$, respectively; Table 2). In the 6 months after initiating GLP-1 RA therapy, the use of oral antidiabetic medication decreased to $85.3 \%$ of patients, with most patients receiving 1 or 2 classes of antidiabetic $(33.0 \%$ and $33.5 \%$, respectively). Prescribing decreased for all classes of oral and injectable antidiabetic medications during the post-index period (Fig. 5a). The largest change was in DPP-4 inhibitors, which were the most commonly prescribed antidiabetic class during the pre-index period (74.1\%) and decreased in usage to $9.0 \%$ after GLP-1 RA therapy initiation (Table $S 1$ in the Electronic supplementary material, ESM). The decrease in prescribing after GLP-1 RA therapy initiation was generally consistent across all other antidiabetic medication classes. Biguanides were the second most commonly prescribed antidiabetic before the index date and the most common after the index date. In the pre-index period, $24.8 \%$ of dulaglutide patients and $11.1 \%$ of liraglutide patients were receiving $\geq 4$ classes of oral antidiabetic medication (Table 2). Nevertheless, a large decrease in DPP-4 inhibitor use and small decreases in the use of all other oral and injectable antidiabetic classes were seen in the dulaglutide and liraglutide groups (Fig. 5b and c), which were consistent with the overall GLP-1 RA population.

\section{Non-antidiabetic Medication}

The use of non-antidiabetic medication remained consistent after the initiation of GLP1 RA therapy (Table S2 in the ESM). Antihypertensive medication was used by approximately half of the patients in the GLP-1 RA group during the pre-index and post-index periods; angiotensin II receptor blockers and calcium channel blockers were the most commonly prescribed antihypertensive medications (Table S2 in the ESM). A lipid-lowering medication was also used by approximately half of patients in the pre-index and post-index periods, with statins being the most commonly prescribed of these medications. Overall, prescribing of non-antidiabetic medication in the dulaglutide and liraglutide groups was consistent with that in the GLP-1 RA population.

\section{GLP-1 RA Treatment Persistence and Modification}

At 6 months post-index, $80.5 \%$ (95\% confidence interval [CI] 77.8-83.0\%) of patients persisted with their index GLP-1 RA therapy in the GLP-1 RA population, including $10.2 \%$ of patients whose index GLP-1 RA therapy was augmented (Table 3). Persistence rates at 12 and 18 months post-index were $66.2 \%$ and $51.6 \%$, respectively. The median duration of persistence until discontinuation or switch during the overall follow-up was 600 days. At all time points, most patients who no longer persisted with their index GLP-1 RA had discontinued, while a minority had switched to a new antidiabetic medication (Table 3).

Persistence rates were $81.9 \% \quad(95 \%$ CI $78.6-84.8 \%)$ in the dulaglutide group and $79.7 \%$ (95\% CI $74.4-84.3 \%$ ) in the liraglutide group at 6 months after initiation of therapy (Table 3). At 12 and 18 months, respectively, $70.7 \%$ and $65.4 \%$ of patients were persisting on their index dulaglutide therapy, and $60.0 \%$ and $30.4 \%$ of patients were persisting on liraglutide over the same period (Table 3; Fig. 6). The median duration of persistence for liraglutide was 440 days. Median duration of persistence could not be calculated for dulaglutide, as greater than $50 \%$ of patients continued their index therapy over the follow-up period.

\section{DISCUSSION}

This is the first known analysis of GLP-1 RA prescribing patterns in Japanese patients with T2DM using the CoDiC database of the JDDM. On average, patients initiated on GLP-1 RA therapy were over 60 years of age, had been diagnosed with T2DM for more than 16 years, were characterized by comorbid obesity, 
(a)

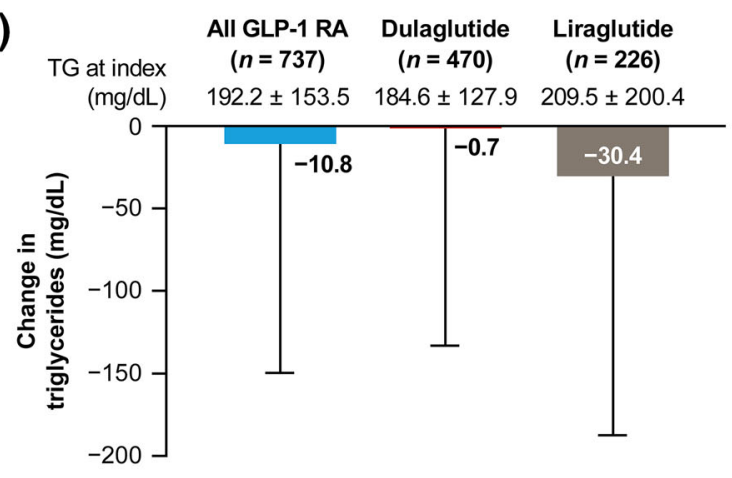

(c)

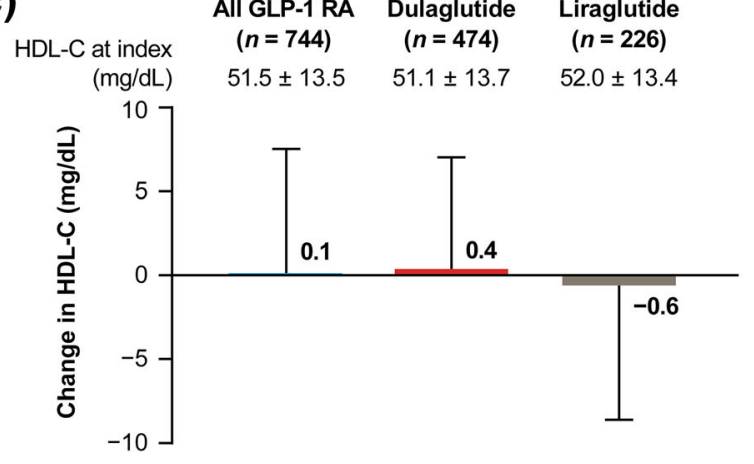

(e)

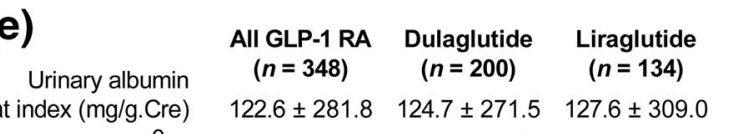

(b) TC at index
$(\mathrm{mg} / \mathrm{dL})$ All GLP-1 RA ( $n=573$ )

Dulaglutide ( $n=334)$ $188.6 \pm 36.2 \quad 188.1 \pm 34.8$

Liraglutide

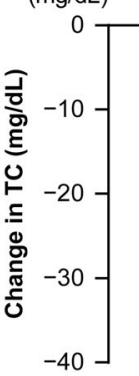

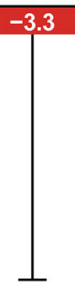

(d) LDL-C at index (mg/dL)

All GLP-1 RA ( $n=595)$ $103.8 \pm 29.7$

Dulaglutide ( $n=383$ )

$186.3 \pm 36.7$

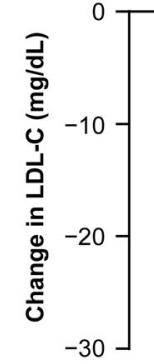

(f)
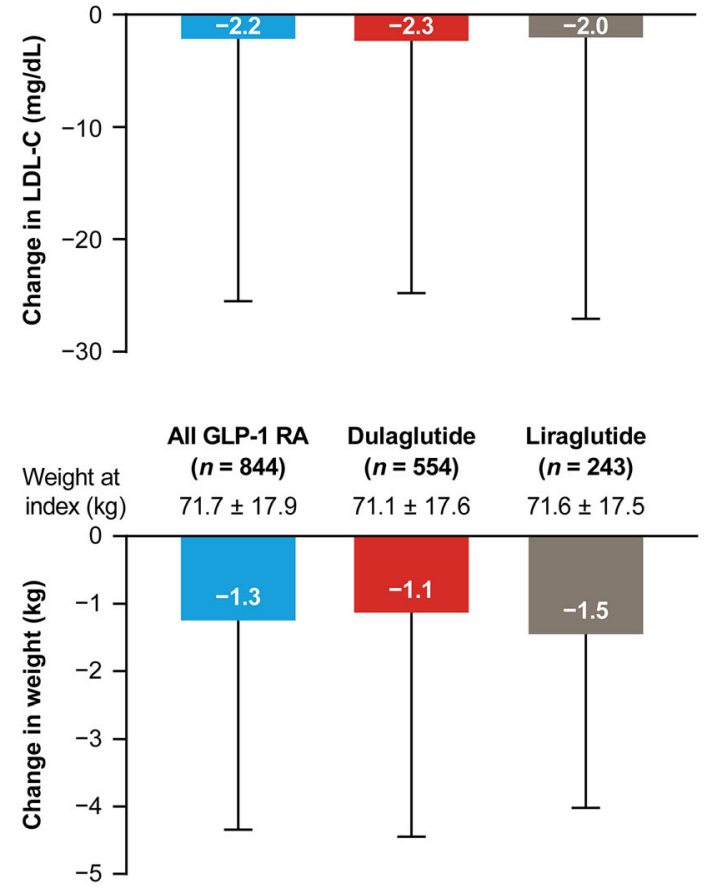

Liraglutide

( $n=182$ )

$97.5 \pm 31.2$

(1) 1 RA Dulaglutide (g)

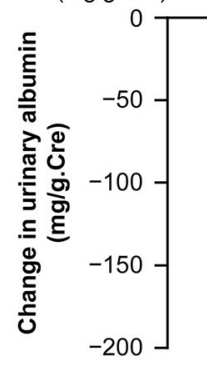

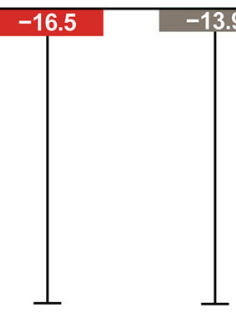

BMl at index $\left(\mathrm{kg} / \mathrm{m}^{2}\right)$

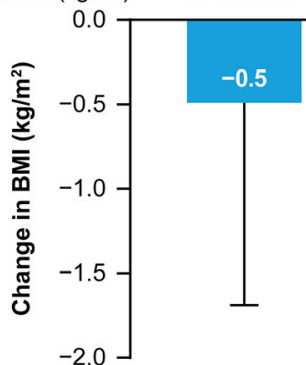

All GLP-1 RA Dulaglutide Liraglutide

$(n=831) \quad(n=545) \quad(n=239)$

( $n=831$ )

$27.5 \pm 5.4$

$26.8 \pm 5.3$

$-0.6$

\section{$-0.5$}

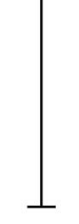


4Fig. 4 Change from index date to 6 months post-index for the GLP-1 RA population in mean a TG, b TC, c HDL-C, d LDL-C, e urinary albumin adjusted for creatinine, $\mathbf{f}$ weight, and $\mathbf{g}$ body mass index. Data are mean \pm standard deviation. BMI body mass index, GLP-I $R A$ glucagon-like peptide- 1 receptor agonist, $H D L-C$ highdensity lipoprotein cholesterol, $L D L-C$ low-density lipoprotein cholesterol, $T C$ total cholesterol, $T G$ triglycerides

hypertension, and dyslipidemia, and were failing to meet glycemic targets despite receiving multiple antidiabetic therapies. Improvements in mean HbA1c and in the proportion of patients achieving target $\mathrm{HbA} 1 \mathrm{c}<7.0 \%$ were seen by 6 months after GLP-1 RA initiation, and were confirmed in the subgroup of patients who continued their index GLP-1 RA throughout the 6-month follow-up. Improvements were also seen in mean body weight and BMI, coupled with decreases in mean TC, TG, LDL-C, and adjusted urinary albumin. Approximately twothirds of patients were prescribed dulaglutide as their first GLP-1 RA, and almost one-third were prescribed liraglutide. Because less than $5 \%$ of patients were prescribed each of the other GLP1 RAs, subgroup analyses for these patients were not feasible. Over $80 \%$ of patients persisted with GLP-1 RA index therapy at 6 months, and persistence rates remained high with dulaglutide at 12 and 18 months. These results illustrate the health outcome benefits of GLP-1 RA therapy in
T2DM and the high persistence achieved with dulaglutide in real-world clinical practice in Japan.

Consistent with our findings in a Japanese population, the tendency to prescribe GLP-1 RA therapy in patients with obesity and poorly controlled diabetes has been reported in a European study of patients with T2DM [17]. In addition, similarly high incidences of comorbid hypertension and dyslipidemia, and the common use of concomitant antihypertensive and lipid-lowering medications, have been identified in patients initiating GLP-1 RA in US and European populations [18, 19]. However, patients in European and US studies initiated GLP-1 RAs at a younger mean age $[17,18,20]$ and after a shorter duration of diabetes [17] than in our Japanese study. The decrease in mean HbA1c levels at 6 months in our study $(0.45 \%)$ was slightly smaller than in real-world analyses from the US, which have shown decreases of $0.90 \%$ at 6 months after initiating dulaglutide and $0.52 \%$ at 12 months after initiating GLP-1 RA therapy in patients with T2DM $[21,22]$. This may be because DPP-4 inhibitors, which have a similar basis of action to GLP-1 RAs [23], are more commonly used in Japan than in the US and Europe [5, 6], and most Japanese patients in our study stopped DPP-4 inhibitor use when initiating GLP-1 RA therapy. The smaller decreases in HbA1c seen in realworld data compared with clinical trials have been associated with poorer adherence to

Table 2 Number of classes of oral antidiabetic medications prescribed in the GLP-1 RA populations

\begin{tabular}{|c|c|c|c|c|c|c|}
\hline \multirow[t]{2}{*}{ Variable } & \multicolumn{2}{|c|}{ All GLP-1 RA $(n=932)$} & \multicolumn{2}{|c|}{ Dulaglutide $(n=612)$} & \multicolumn{2}{|c|}{ Liraglutide $(n=271)$} \\
\hline & $\begin{array}{l}6 \text { months pre- } \\
\text { index }\end{array}$ & $\begin{array}{l}6 \text { months post- } \\
\text { index }\end{array}$ & $\begin{array}{l}6 \text { months pre- } \\
\text { index }\end{array}$ & $\begin{array}{l}6 \text { months post- } \\
\text { index }\end{array}$ & $\begin{array}{l}6 \text { months pre- } \\
\text { index }\end{array}$ & $\begin{array}{l}6 \text { months post- } \\
\text { index }\end{array}$ \\
\hline \multicolumn{7}{|c|}{ Oral antidiabetic medication classes, $n(\%)$} \\
\hline 0 & $56(6.0)$ & $137(14.7)$ & $31(5.1)$ & $76(12.4)$ & $22(8.1)$ & $54(19.9)$ \\
\hline 1 & $174(18.7)$ & $308(33.0)$ & $89(14.5)$ & $192(31.4)$ & $68(25.1)$ & $96(35.4)$ \\
\hline 2 & $251(26.9)$ & $312(33.5)$ & $157(25.7)$ & $211(34.5)$ & $77(28.4)$ & $86(31.7)$ \\
\hline 3 & $266(28.5)$ & $143(15.3)$ & $183(29.9)$ & $107(17.5)$ & $74(27.3)$ & $29(10.7)$ \\
\hline$\geq 4$ & $185(19.8)$ & $32(3.4)$ & $152(24.8)$ & $26(4.2)$ & $30(11.1)$ & $6(2.2)$ \\
\hline
\end{tabular}

GLP-1 RA glucagon-like peptide-1 receptor agonist 
(a)

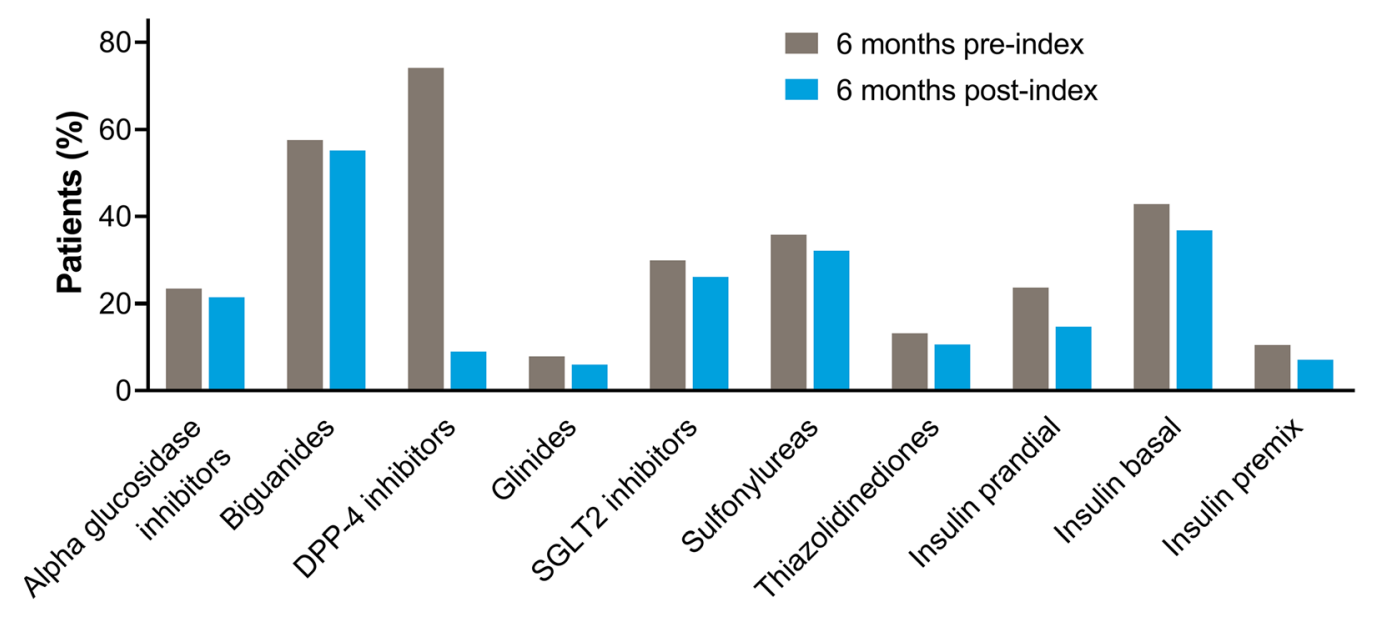

(b)

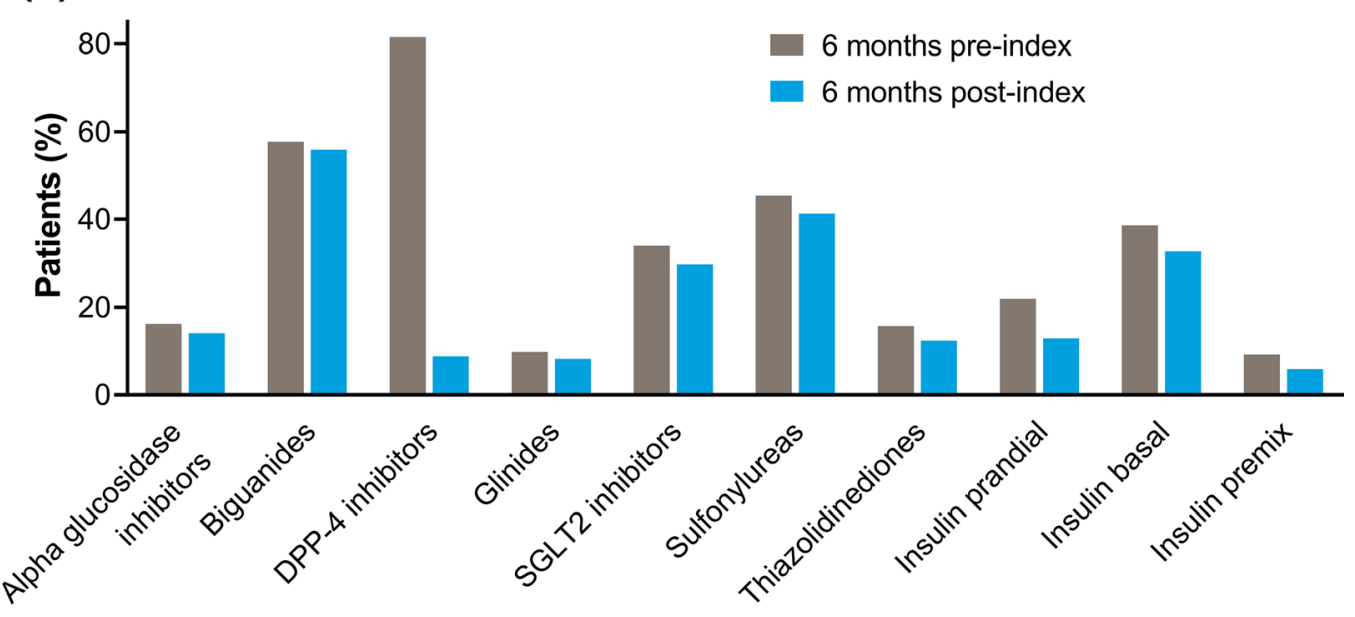

(c)
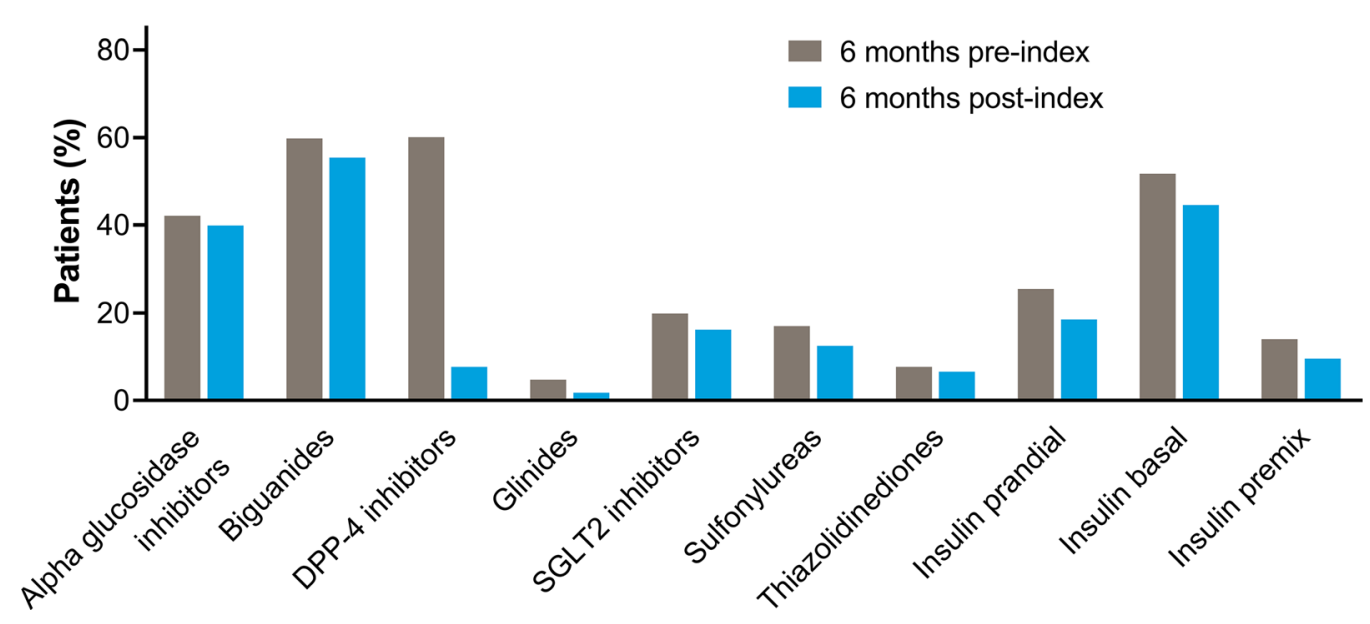
4Fig. 5 Antidiabetic medications prescribed during 6 months pre-index and 6 months post-index for a the GLP-1 RA population, b patients with an index GLP-1 RA of dulaglutide, and $\mathbf{c}$ patients with an index GLP-1 RA of liraglutide. DPP-4 dipeptidyl peptidase-4, GLP-1 RA glucagon-like peptide-1 receptor agonist, $S G L T 2$ sodiumglucose transport protein 2

diabetes medication in clinical practice than in a trial setting [22]. Although we were unable to assess HbA1c at 12 and 18 months in our study, it is likely that reductions in $\mathrm{HbA1c}$ would have continued while patients persisted on their medication, as seen in non-Japanese studies $[18,22]$. Reported 12-month GLP-1 RA persistence rates of $63.6-70.5 \%$ (reported as nonpersistence rates of 29.5-36.4\%) in Europe [24] and $51.9 \%$ in the US [25] are consistent with the GLP-1 RA persistence rate of $66.2 \%$ seen in our study at 12 months, despite the eligibility requirement of $\geq 6$ months post-index follow-

Table 3 Index GLP-1 RA persistence and first modification at 6 months post-index and during overall follow-up

\begin{tabular}{|c|c|c|c|}
\hline Variable & $\begin{array}{l}\text { All GLP-1 RA } \\
(n=932)\end{array}$ & $\begin{array}{l}\text { Dulaglutide } \\
(n=612)\end{array}$ & $\begin{array}{l}\text { Liraglutide } \\
(n=271)\end{array}$ \\
\hline \multicolumn{4}{|l|}{ Persistence ${ }^{\mathrm{a}}, \%(95 \%$ CI $)$} \\
\hline 6 months & $80.5(77.8-83.0)$ & $81.9(78.6-84.8)$ & $79.7(74.4-84.3)$ \\
\hline 12 months & $66.2(63.1-69.5)$ & $70.7(67.0-74.6)$ & $60.0(54.2-66.4)$ \\
\hline 18 months & $51.6(47.7-55.9)$ & $65.4(61.0-70.1)$ & $30.4(24.3-38.1)$ \\
\hline $\begin{array}{l}\text { Duration of persistence }{ }^{\mathrm{a}} \text {, days, median }(95 \% \\
\text { CI) }\end{array}$ & $600(526.0-\mathrm{NC})$ & NC & $440(396.0-484.0)$ \\
\hline \multicolumn{4}{|l|}{ Any first index treatment modification ${ }^{\mathrm{b}}, n(\%)$} \\
\hline 6 months & $274(29.4)$ & $173(28.3)$ & $79(29.2)$ \\
\hline Overall follow-up & $507(54.4)$ & $293(47.9)$ & $183(67.5)$ \\
\hline \multicolumn{4}{|l|}{ First discontinuation ${ }^{\mathrm{c}}, n(\%)$} \\
\hline 6 months & $148(15.9)$ & $94(15.4)$ & $43(15.9)$ \\
\hline Overall follow-up & $279(29.9)$ & $145(23.7)$ & $118(43.5)$ \\
\hline \multicolumn{4}{|l|}{ First switch ${ }^{\mathrm{d}}, n(\%)$} \\
\hline 6 months & $32(3.4)$ & $16(2.6)$ & $11(4.1)$ \\
\hline Overall follow-up & $50(5.4)$ & $22(3.6)$ & $21(7.7)$ \\
\hline \multicolumn{4}{|l|}{ First augmentation $^{\mathrm{e}}, n(\%)$} \\
\hline 6 months & $95(10.2)$ & $64(10.5)$ & $25(9.2)$ \\
\hline Overall follow-up & $179(19.2)$ & $127(20.8)$ & $44(16.2)$ \\
\hline
\end{tabular}

$C I$ confidence interval, $N C$ not calculable, GLP-1 RA glucagon-like peptide-1 receptor agonist

${ }^{a}$ Persistence was the duration of index GLP-1 RA therapy from initiation to discontinuation or switch

${ }^{b}$ Any first index GLP-1 RA treatment modification included the first post-index discontinuation, switch, or augmentation

${ }^{c}$ Discontinuation was an absence of any prescription for the index GLP-1 RA for $>60$ days since the previous visit

${ }^{d}$ Switch was the prescription of a new antidiabetic medication within 30 days before or after discontinuation of the index GLP-1 RA

e Augmentation was the prescription of a new antidiabetic medication $>30$ days before index GLP-1 RA discontinuation or the end of the follow-up period, whichever came first 


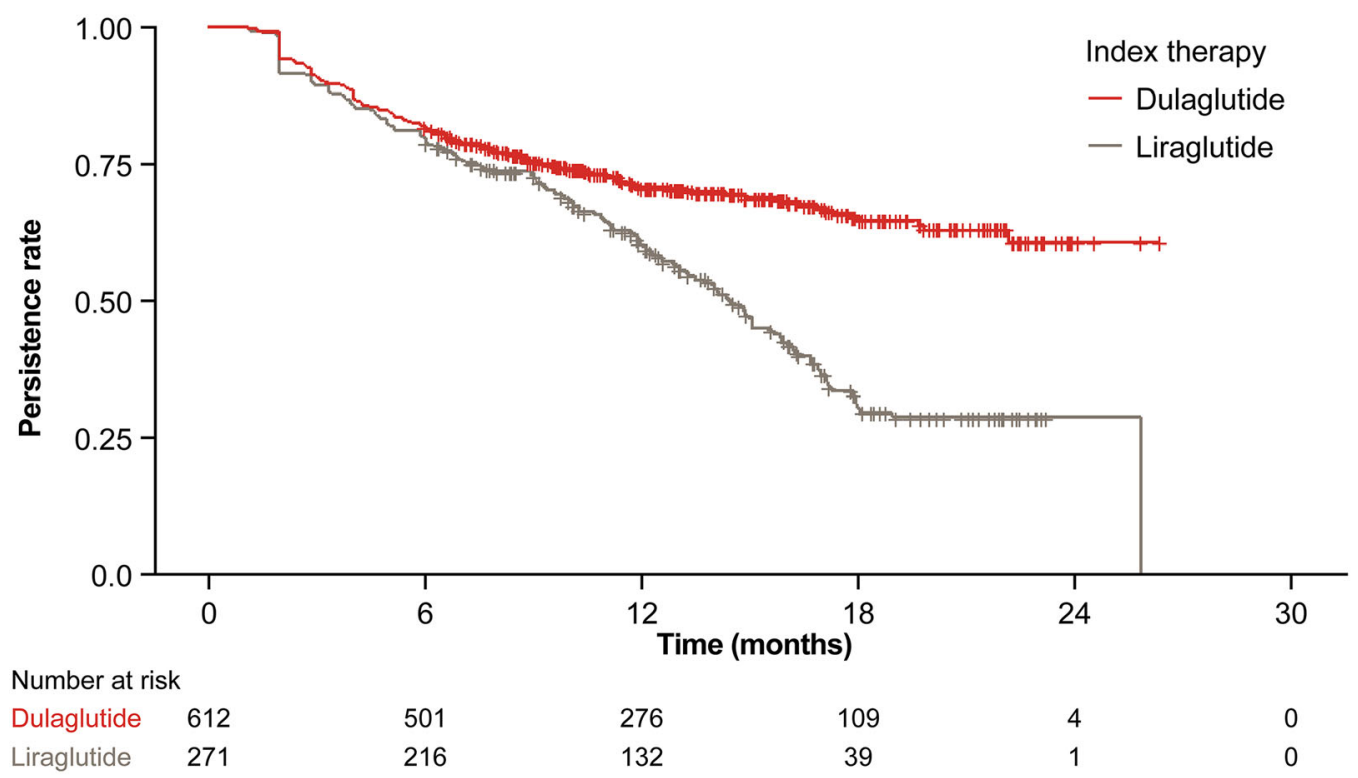

Fig. 6 Kaplan-Meier analysis of GLP-1 RA persistence during the overall follow-up period. GLP-1 RA glucagon-like peptide-1 receptor agonist

up in our study versus $\geq 12$ months in the European and US studies.

In this study, initiation of any GLP-1 RA therapy was associated with a decrease in the number of concomitant antidiabetic medications, suggesting that GLP-1 RAs are being prescribed as a replacement rather than an addition to existing antidiabetic therapy. Concomitant DPP-4 inhibitor use decreased dramatically after initiation of GLP-1 RA therapy. Some prefectures in Japan allow reimbursement for concomitant use of DPP-4 inhibitors during GLP-1 RA therapy; however, the $9 \%$ of patients prescribed concomitant DPP-4 inhibitors in this study may include patients who switched from GLP-1 RA to DPP-4 inhibitor therapy but whose discontinuation of GLP-1 RA therapy was not immediately reflected in prescribing data, which is a recognized limitation of prescription data analyses. All other oral antidiabetic medications and insulins showed smaller, consistent decreases in prescribing post-index. As GLP-1 RA therapy is not recommended in patients with poorly controlled HbA1c using multiple oral antidiabetic medications, early initiation of GLP-1 RAs is advised.

GLP-1 RAs have been shown to reduce body weight by slowing gastric emptying and inducing satiety [10-12]. Obesity was a common comorbidity in GLP-1 RA initiators, and both dulaglutide and liraglutide were associated with reduced body weight and BMI at 6 months post-index, despite neither treatment significantly decreasing body weight in Japanese patients with T2DM in a clinical trial setting $[26,27]$. An earlier study in Japan indicated that diabetes specialists choose GLP-1 RAs for obese patients with T2DM, suggesting that concerns over weight loss as well as glycemic control are driving prescribing decisions [9]. This reflects findings in other countries of high BMI in patients initiating GLP-1 RAs [17, 20].

Interpretation of the findings from this study should take into account the retrospective nature of the study design, lack of comparative analyses, and strengths and limitations of the CoDiC database. First, the database includes a large number of patients and reflects a realworld T2DM population in Japan, but because patients in the database are treated by diabetes specialists, and predominantly at clinics rather than hospitals, the findings from this study may not be representative of the entire population. Additionally, data collection is per institution, so patient follow-up is incomplete if patients are transferred from JDDM institutions to 
occasionally use non-JDDM institutions. However, as GLP-1 RA therapy is generally initiated at specialist clinics in Japan, the coverage of the database can likely be considered representative of new users of GLP-1 RA therapy. Second, the reliability of the data in the CoDiC database is likely to be high because the data are entered by JDDM members and physicians from specialized diabetes institutions according to JDDM guidelines. Moreover, as both patient and laboratory pathology data are available from the database, assessments of the relationships between prescription patterns and clinical outcomes can be conducted. However, in this study, laboratory data were not analyzed for patients who persisted beyond 6 months, and no analyses stratified by GLP-1 RA were performed. Finally, the overall GLP-1 RA population at 6 months included approximately $20 \%$ of patients who had discontinued GLP-1 RA therapy, and the reasons for their GLP-1 RA discontinuation could not be assessed; however, the subgroup analysis conducted in patients who continued their index GLP-1 RA for at least 6 months showed similar results for change in HbA1c compared with the overall population. Therefore, it is likely that the patients who had discontinued therapy in the overall population did not have a major impact on the clinical outcomes. Since the end of the data collection period for this study, the subcutaneous GLP-1 RA semaglutide and a higher dose of liraglutide have been approved in Japan. Future updates to this study will be important to understand the evolution of real-world prescribing practices.

\section{CONCLUSION}

This is the first study to define the demographic and clinical characteristics of patients with T2DM initiating GLP-1 RA therapy in Japan using the CoDiC database of the JDDM. The results illustrate the real-world health outcome benefits of GLP-1 RA therapy, including improvements in $\mathrm{HbA1c}$, body weight, and blood lipid profile. The study also supports the high rates of long-term persistence previously reported for dulaglutide, the GLP-1 RA most commonly prescribed for T2DM in Japanese clinical practice.

\section{ACKNOWLEDGEMENTS}

We thank all of the physicians involved in the JDDM Study Group.

Funding. Sponsorship for this study and the Rapid Service Fee were funded by Eli Lilly Pty. Ltd., Kobe-shi, Japan.

Authorship. All named authors meet the International Committee of Medical Journal Editors (ICMJE) criteria for authorship for this article, take responsibility for the integrity of the work as a whole, and have given their approval for this version to be published.

Authorship Contributions. All authors were involved in the study design and the interpretation of study results, and participated in the drafting, critical revision, and approval of the final version of the manuscript. Yasushi Ishigaki and Hiroshi Maegawa were investigators and involved in the data collection. Alena Strizek, Zhihong Cai, and Nobuhiro Arai conducted the statistical analysis.

Medical Writing, Editorial, and Other Assistance. Medical writing assistance was provided by Linda Donnini, PhD, CMPP and Serina Stretton, PhD, CMPP of ProScribe-Envision Pharma Group, and was funded by Eli Lilly and Company. ProScribe's services complied with international guidelines for Good Publication Practice (GPP3).

Disclosures. Yasushi Ishigaki has received lecture fees from Bayer Yakuhin, Kowa Pharmaceutical Company, MSD, Novartis, Novo Nordisk, Ono Pharmaceutical, Sanofi K.K., and Takeda Pharmaceutical; scholarship donations from MSD and Ono Pharmaceutical; and research funding from Daiichi Sankyo Company and Takeda Science Foundation.

Alena Strizek was an employee of Eli Lilly and Company during the period of the study and is currently an employee of Amgen. Toshihiko 
Aranishi, Takeshi Imaoka, and Zhihong Cai are employees of Eli Lilly Japan K.K. Takeshi Imaoka and Zhihong Cai are shareholders of Eli Lilly and Company, and Toshihiko Aranishi is a shareholder of Chugai Pharmaceutical Co., Ltd. Nobuhiro Arai was an employee of Deloitte Tohmatsu Consulting LLC during the period of the study and is currently an employee of Marushin Consulting K.K., Tokyo, Japan.

Hiroshi Maegawa has received lecture fees from Astellas Pharma, Astra Zeneca, Boehringer Ingelheim, Daiichi Sankyo Company, Eli Lilly and Company, Mitsubishi Tanabe Pharma Corporation, MSD, Novo Nordisk, Sanofi, Sumitomo Dainippon Pharma, and Takeda Pharmaceutical; scholarship donations from Astellas Pharma, Bayer Yakuhin, Boehringer Ingelheim, Daiichi Sankyo Company, Eli Lilly and Company, Kowa Pharmaceutical Company, Mitsubishi Tanabe Pharma Corporation, MSD, Nipro Corporation, Novartis, Novo Nordisk, Ono Pharmaceutical, Sanofi, Sanwa Kagaku Kenkyusho, Shionogi \& Co., Sumitomo Dainippon Pharma, Taisho Pharma, Takeda Pharmaceutical, and Teijin; and research funding from Astellas Pharma, Astra Zeneca, Boehringer Ingelheim, CMIC, Kyowa Kirin, MIKI Corporation, Mitsubishi Tanabe Pharma Corporation, Nissan Chemical Corporation, and Sunstar.

Compliance with Ethics Guidelines. The study protocol was approved by the JDDM Ethical Review Board (JDDM2018-2). All patients provided informed consent. The study was conducted in accordance with the ethical principles of the Declaration of Helsinki 1964 and its later amendments, Good Pharmacoepidemiology Practices, and applicable local laws and regulations.

Data Availability. The datasets analyzed during the current study are not publicly available because they are sampled from the CoDiC database, but are available from Nobuhiro Arai on reasonable request.

Open Access. This article is licensed under a Creative Commons Attribution-NonCommercial 4.0 International License, which permits any non-commercial use, sharing, adaptation, distribution and reproduction in any medium or format, as long as you give appropriate credit to the original author(s) and the source, provide a link to the Creative Commons licence, and indicate if changes were made. The images or other third party material in this article are included in the article's Creative Commons licence, unless indicated otherwise in a credit line to the material. If material is not included in the article's Creative Commons licence and your intended use is not permitted by statutory regulation or exceeds the permitted use, you will need to obtain permission directly from the copyright holder. To view a copy of this licence, visit http:// creativecommons.org/licenses/by-nc/4.0/.

\section{REFERENCES}

1. Japan Ministry of Health, Labour and Welfare. National Health and Nutrition Survey (NHNS), 2017. Available at: https://www.mhlw.go.jp/ content/000451755.pdf. Accessed 10 Feb 2020.

2. Morimoto A, Nishimura R, Tajima N. Trends in the epidemiology of patients with diabetes in Japan. JMAJ. 2010;53:36-40.

3. Haneda $M$, Noda $M$, Origasa $H$, et al. Japanese clinical practice guideline for diabetes 2016. J Diabetes Investig. 2018;9:657-97.

4. Buse JB, Wexler DJ, Tsapas A, et al. Update to: Management of hyperglycemia in type 2 diabetes, 2018. A consensus report by the American Diabetes Association (ADA) and the European Association for the Study of Diabetes (EASD). Diabetes Care. 2019;43:487-93.

5. Seino Y, Kuwata H, Yabe D. Incretin-based drugs for type 2 diabetes: focus on East Asian perspectives. J Diabetes Investig. 2016;7(Suppl 1):102-9.

6. Kubota K, Kamijima Y, Kao Yang YH, et al. Penetration of new antidiabetic medications in East Asian countries and the United States: a cross-national comparative study. PLoS ONE. 2018;13: e0208796.

7. Fukushima M, Suzuki H, Seino Y. Insulin secretion capacity in the development from normal glucose tolerance to type 2 diabetes. Diabetes Res Clin Pract. 2004;66(Suppl 1):S37-43. 
8. Miyazawa I, Kadota A, Miura K, et al. Twelve-year trends of increasing overweight and obesity in patients with diabetes: the Shiga Diabetes Clinical Survey. Endocr J. 2018;65:527-36.

9. Fujihara K, Hanyu O, Heianza Y, et al. Comparison of clinical characteristics in patients with type 2 diabetes among whom different antihyperglycemic agents were prescribed as monotherapy or combination therapy by diabetes specialists. J Diabetes Investig. 2016;7:260-9.

10. Drucker DJ, Nauck MA. The incretin system: glucagon-like peptide-1 receptor agonists and dipeptidyl peptidase- 4 inhibitors in type 2 diabetes. Lancet. 2006;368:1696-705.

11. Koliaki C, Doupis J. Incretin-based therapy: a powerful and promising weapon in the treatment of type 2 diabetes mellitus. Diabetes Ther. 2011;2: $101-21$.

12. Ross SA, Ballantine J. Early use of glucagon-like peptide-1 receptor agonists (GLP-1 RAs) in type 2 diabetes. Curr Med Res Opin. 2013;29:1617-26.

13. Kim YG, Hahn S, Oh TJ, Park KS, Cho YM. Differences in the HbA1c-lowering efficacy of glucagonlike peptide- 1 analogues between Asians and nonAsians: a systematic review and meta-analysis. Diabetes Obes Metab. 2014;16:900-9.

14. Nishimura R, Kato H, Kisanuki K, et al. Treatment patterns, persistence and adherence rates in patients with type 2 diabetes mellitus in Japan: a claims-based cohort study. BMJ Open. 2019;9: e025806.

15. Kobayashi M, Yamazaki K, Hirao K, et al. The status of diabetes control and antidiabetic drug therapy in Japan-a cross-sectional survey of 17,000 patients with diabetes mellitus (JDDM 1). Diabetes Res Clin Pract. 2006;73:198-204.

16. Sekimoto M, Ii M. Supplier-induced demand for chronic disease care in Japan: multilevel analysis of the association between physician density and physician-patient encounter frequency. Value Health Reg Issues. 2015;6:103-10.

17. Tofé S, Argüelles I, Mena E, et al. Real-world GLP-1 RA therapy in type 2 diabetes: a long-term effectiveness observational study. Endocrinol Diabetes Metab. 2018;2:e00051.

18. Mody R, Huang Q, Yu M, et al. Adherence, persistence, glycaemic control and costs among patients with type 2 diabetes initiating dulaglutide compared with liraglutide or exenatide once weekly at 12-month follow-up in a real-world setting in the United States. Diabetes Obes Metab. 2019;21:920-9.

19. Federici MO, McQuillan J, Biricolti G, et al. Utilization patterns of glucagon-like peptide-1 receptor agonists in patients with type 2 diabetes mellitus in Italy: a retrospective cohort study. Diabetes Ther. 2018;9:789-801.

20. Yu M, Mody R, Landó LF, et al. Characteristics associated with the choice of first injectable therapy among US patients with type 2 diabetes. Clin Ther. 2017;39:2399-408.

21. Mody R, Grabner M, Yu M, et al. Real-world effectiveness, adherence and persistence among patients with type 2 diabetes mellitus initiating dulaglutide treatment. Curr Med Res Opin. 2018;34:995-1003.

22. Carls GS, Tuttle E, Tan RD, et al. Understanding the gap between efficacy in randomized controlled trials and effectiveness in real-world use of GLP-1 RA and DPP-4 therapies in patients with type 2 diabetes. Diabetes Care. 2017;40:1469-78.

23. Spellman CW. Pharmacology of GLP-1 agonists: describing the therapeutic potential to patients. J Am Osteopath Assoc. 2011;111:eS10-4.

24. Wilke T, Mueller S, Groth A, et al. Non-persistence and non-adherence of patients with type 2 diabetes mellitus in therapy with GLP-1 receptor agonists: a retrospective analysis. Diabetes Ther. 2016;7: $105-24$.

25. Cai J, Divino V, Burudpakdee C. Adherence and persistence in patients with type 2 diabetes mellitus newly initiating canagliflozin, dapagliflozin, DPP$4 \mathrm{~s}$, or GLP-1s in the United States. Curr Med Res Opin. 2017;33:1317-28.

26. Miyagawa J, Odawara M, Takamura T, Iwamoto N, Takita Y, Imaoka T. Once-weekly glucagon-like peptide-1 receptor agonist dulaglutide is non-inferior to once-daily liraglutide and superior to placebo in Japanese patients with type 2 diabetes: a 26-week randomized phase III study. Diabetes Obes Metab. 2015;17:974-83.

27. Odawara M, Miyagawa J, Iwamoto N, Takita Y, Imaoka T, Takamura T. Once-weekly glucagon-like peptide-1 receptor agonist dulaglutide significantly decreases glycated haemoglobin compared with once-daily liraglutide in Japanese patients with type 2 diabetes: 52 weeks of treatment in a randomized phase III study. Diabetes Obes Metab. 2016;18: 249-57. 